\title{
INTEGRATION ON NONCOMPACT SUPERMANIFOLDS
}

\author{
MITCHELL J. ROTHSTEIN
}

\begin{abstract}
ABSTRACr. We note that the Berezin integral, which is ill-defined for noncompact supermanifolds, is a distribution with support on the underlying manifold. This leads to the discovery of correction terms in the Berezinian transformation law and thereby eliminates the boundary ambiguities.
\end{abstract}

1. Introduction. A perspective on the Berezin integral. A real $(p, q)$-dimensional $C^{\infty}$ supermanifold is a ringed space $(M, \mathscr{A})$ such that

(1.1) $\mathscr{A}$ is a sheaf of supercommutative algebras over $\mathbf{R}$.

(1.2) $(M, \mathscr{A} / \mathscr{N})$ is a $p$-dimensional $C^{\infty}$ manifold, where $\mathscr{N}$ is the sheaf of nilpotents. We denote $\mathscr{A} / \mathscr{N}$ by $C^{\infty}$.

(1.3) For any point $m \in M$, there is a neighborhood $\mathscr{U}$ of $m$ such that $\left.\mathscr{A}\right|_{\mathscr{U}} \approx$ $\left.C^{\infty}\right|_{\mathscr{U}} \otimes \Lambda\left(\mathbf{R}^{q}\right)$ as sheaves of supercommutative algebras.

One may imagine that $M$ sits inside some larger manifold and that $\mathscr{A}$ is the restriction of the structure sheaf of the larger manifold to $M$. The ideal in $\mathscr{A}$ defining $M$ as a submanifold is generated by coordinates which anticommute.

An integration theory in the category of supermanifolds should consist of the prescription of a suitable sheaf of $\mathscr{A}$ modules, which may be called volume forms, and a linear functional on its global sections. There is a first candidate for such a sheaf: the $p$-forms on $M$ form a $C^{\infty}$ module and therefore also an $\mathscr{A}$ module, since $C^{\infty}=\mathscr{A} / \mathscr{N}$. Since this ignores the anticommuting coordinates it is an uninteresting choice. But if one regards a $p$-form on $M$ as a distribution on $\mathscr{A}$ supported on $M$, specifically one which annihilates the ideal defining $M$, then one is led to consider more general distributions, namely those of the form

$$
f \mapsto \int_{M} \omega \cdot L[f]
$$

where $\omega$ is a $p$-form on $M$ and $L$ is a differential operator on $\mathscr{A}$. In general, $L$ will involve differentiation with respect to the anticommuting coordinates, so that for any section $f \in \Gamma(\mathscr{A})$, nilpotent or not, there will be some distribution of the form (1.4) for which $\int_{M} \omega \cdot L[f] \neq 0$. This suggests that a better candidate for the volume sheaf of $\mathscr{A}$ is $\Omega_{M} \otimes_{\mathscr{A}} \mathscr{D}$, the sheaf of linear differential operators on $\mathscr{A}$ whose values are $p$-forms on $M$. ( $\mathscr{D}$ stands for the sheaf of differential operators on $\mathscr{A}$.) If

Received by the editors December 21, 1985.

1980 Mathematics Subject Classification (1985 Revision). Primary 58C50.

Supported in part by NSF Grant No. DMS-8501783.

(C)1987 American Mathematical Society $0002-9947 / 87 \$ 1.00+\$ .25$ per page 
$M$ is oriented, a section $\omega \in \Gamma\left(\Omega^{p} \otimes \mathscr{D}\right)$ defines a linear functional

$$
\Gamma(\mathscr{A}) \rightarrow \mathbf{R},\langle\omega, f\rangle=\int_{M} \omega[f],
$$

provided this converges. $\Omega^{p} \otimes \mathscr{D}$ is naturally a right $\mathscr{D}$-module, and $\langle\omega, f\rangle$ $=\langle\omega f, 1\rangle$, so the natural definition for the integral on sections of $\Omega^{p} \otimes \mathscr{D}$ is

$$
\int_{\mathscr{A}} \omega=\int_{M} \omega[1]
$$

The point that will be developed in this paper is that this is the Berezin integral. More precisely, there is a codimension-one subsheaf $\left(\Omega^{p} \otimes \mathscr{D}\right)^{+} \subset \Omega^{p} \otimes \mathscr{D}$, to be defined in $\S 2$, such that if $\omega$ is any global section of $\left(\Omega^{p} \otimes \mathscr{D}\right)^{+}$and $f$ is any global section of $\mathscr{A}$, then $\omega[f]$ is an exact $p$-form. Therefore on compact supermanifolds without boundary the integral $(1.5)$ is well defined on sections of $\Omega^{p} \otimes$ $\mathscr{D} /\left(\Omega^{p} \otimes \mathscr{D}\right)^{+}$. This quotient turns out to be the sheaf of Berezin forms, known as $\mathscr{B}$ er, and (1.5) yields the Berezin integration formula. (For a brief introduction to Berezin integration see $\$ \S 2,3$. A better introduction may be found in [3], and a beautiful elaboration of $\mathscr{B} e r$ is given in [4].) The sequence

$$
0 \rightarrow\left(\Omega^{p} \otimes \mathscr{D}\right)^{+} \rightarrow \Omega^{p} \otimes \mathscr{D} \rightarrow \mathscr{B} e r \rightarrow 0
$$

does not split naturally, which is why noncompactly supported sections of $\mathscr{B} e r$ cannot be integrated.

Though nilpotent sections of $\mathscr{A}$ move across the $\otimes$ sign as zero, $\Omega^{p} \otimes \mathscr{D}$ and $\left(\Omega^{p} \otimes \mathscr{D}\right)^{+}$are nevertheless free $\mathscr{A}$ modules under right multiplication (Proposition 2.1). By choosing a local splitting of (1.6) and studying the transformation law for $\Omega^{p} \otimes \mathscr{D}$ under change of coordinates, one may see which boundary terms to add in order to compensate for the ambiguity in the Berezin integral. The explicit formula for this transformation law is the main result of $\S 3$.

One may also consider $\Omega_{M}^{k} \otimes_{\mathscr{A}} \mathscr{D}$ for $k<p . \Omega^{\bullet} \otimes \mathscr{A}$ is naturally a complex (similar to one described in [4]), and a version of Stokes' theorem appears automatically. This is described in $\$ 4$.

Other authors have addressed the problem of integration on supermanifolds from a different point of view. See [5, 6]. For general background regarding supermanifolds, see [2, 3].

I wish to thank George Jennings and Robin Graham for their enthusiasm and many helpful conversations.

2. The Fermi integral, the Berezin integral, and the Lie derivative. $\Omega^{p} \otimes \mathscr{A}$ is a left and right $\mathscr{A}$ module. The right action is the good one.

Proposition 2.1. $\Omega^{p} \otimes \mathscr{D}$ is a locally free $\mathscr{A}$ module of infinite rank.

Proof. Given an isomorphism $\left.\left.\mathscr{A}\right|_{\mathscr{U}} \stackrel{\tau}{\rightarrow} C^{\infty}\right|_{\mathscr{U}} \otimes \Lambda(V)$, where $\operatorname{dim} V=q$, and given coordinates $x^{1}, \ldots, x^{p}$ for $\mathscr{U}$ and a basis $\theta^{1}, \ldots, \theta^{q}$ for $V$, the sections $\tau^{-1}\left(x^{1}\right), \ldots, \tau^{-1}\left(x^{p}\right), \tau^{-1}\left(\theta^{1}\right), \ldots, \tau^{-1}\left(\theta^{q}\right)$ are called a coordinate system for $\left.\mathscr{A}\right|_{\mathscr{U}}$. If $X$ is a derivation of $\mathscr{A}$ and $f$ is a section of $\mathscr{A}$, the differential $d f$ is defined by $d f(X)=(-1)^{|X||f|} X[f]$. The partial derivatives are defined by $d \theta^{a}\left(\partial / \partial \theta^{b}\right)=\delta_{b}^{a}$, 
etc. Note that $\partial \theta^{a} / \partial \theta^{b}=-\delta_{b}^{a}$. A general section of $\left.\Omega^{p} \otimes \mathscr{D}\right|_{\mathscr{U}}$ may be written

$$
d x^{1} \cdots d x^{p} \otimes \frac{\partial}{\partial x^{I}} \frac{\partial}{\partial \theta^{\mu}} \circ f^{I, \mu}
$$

where $I=\left(i_{1}, \ldots, i_{p}\right) \in\left(\mathbf{Z}^{+}\right)^{p}$ and $\mu=\left(\mu_{1}, \ldots, \mu_{q}\right) \in\{0,1\}^{q}$. In what follows, the $\otimes$ and $\circ$ will be omitted, with the understanding that a nilpotent function appearing to the left of the derivatives is 0 , and that $\left(\partial / \partial x^{i}\right) f$ means composition of operators. If it is intended that the operator $L$ be applied to the function $f$, the notation $L[f]$ will be used. Also $d x^{1} \cdots d x^{p}$ will be abbreviated to $d x$, and $\partial / \partial \theta^{q} \cdots \partial / \partial \theta^{1}$ will be abbreviated to $\partial_{\theta}$. Now write $f^{I, \mu}=f_{\rho}^{I, \mu}(x) \theta^{\rho}$, where $\rho$ is a multi-index of the same sort as $\mu$. The sum in (2.1) is not free: Because the nilpotents annihilate $d x$, $d x\left(\partial / \partial \theta^{\mu}\right) \theta^{\rho}=0$ unless $\rho<\mu$. But for the same reason, $d x \partial_{\theta} \theta^{\mu}= \pm d x\left(\partial / \partial \theta^{1-\mu}\right)$, where $1-\mu=\left(1-\mu_{1}, \ldots, 1-\mu_{q}\right)$. Therefore the sections $d x\left(\partial / \partial x^{I}\right) \partial_{\theta}$ span $\left.\Omega^{p} \otimes \mathscr{D}\right|_{\mathscr{U}}$, and they are easily seen to be linearly independent.

Given an arbitrary coordinate system $(x, \theta)$, define

$$
D_{I}(x, \theta)=(-1)^{|I|} d x \frac{\partial}{\partial x^{I}} \partial_{\theta} .
$$

By Proposition 2.1, the $D_{I}(x, \theta)$ 's form a local basis for $\Omega^{p} \otimes \mathscr{D}$.

Define the Fermi integral, $\int_{F}$, to be the sheaf morphism

That is,

$$
\Omega^{p} \otimes \mathscr{D} \stackrel{f_{F}}{\rightarrow} \Omega^{p}, \quad \int_{F} \omega=\omega[1]
$$

$$
\int_{F} D_{I}(x, \theta) f^{I}=(-1)^{|I|} d x^{1} \cdots d x^{p} \frac{\partial}{\partial \theta^{q} \cdots \partial \theta^{1}}\left[\frac{\partial f^{I}}{\partial x^{I}}\right] .
$$

If $M$ is oriented, the Berezin integral is the iterated integral

$$
\int_{\text {Ber }} \omega=\int_{M} \int_{F} \omega=\int_{M} \omega[1]
$$

If $f^{I}=0$ for $I>0$, this is the usual definition of the Berezin integral. If, in addition, $p=0$, this is the integration over Grassmann variables introduced by Berezin in [1].

Given an open set $\mathscr{U}$, define $\left(\Omega^{p} \otimes \mathscr{D}\right)^{+}(\mathscr{U}) \subset \Omega^{p} \otimes \mathscr{D}(\mathscr{U})$ by the condition that $\omega \in\left(\Omega^{p} \otimes \mathscr{D}\right)^{+}(\mathscr{U})$ if and only if for any section $h \in \mathscr{A}(M)$ with $\operatorname{supp}(h)$ compact and contained in $\mathscr{U}, \int_{M} \omega[h]=0$. It is an easy exercise that $\mathscr{U} \rightarrow\left(\Omega^{p} \otimes \mathscr{D}\right)^{+}(\mathscr{U})$ is a complete presheaf.

If $(x, \theta)$ is a coordinate system on $\mathscr{U}$ and $h$ is compactly supported inside $\mathscr{U}$, then $\int_{M} d x\left(\partial h / \partial x^{I}\right)=0$ whenever $I>0$. From this follows

Proposition 2.2. Let $(x, \theta)$ be a coordinate system. Take $\omega \in \Omega^{p} \otimes \mathscr{D}$ and write $\omega=D_{I}(x, \theta) f^{I}$. Then $\omega \in\left(\Omega^{p} \otimes \mathscr{D}\right)^{+}$if and only if $f^{0}=0$.

By Propositions 2.1 and 2.2, $\left(\Omega^{p} \otimes \mathscr{D}\right) /\left(\Omega^{p} \otimes \mathscr{D}\right)^{+}$is a locally free $\mathscr{A}$ module of rank 1 , with local generator $D(x, \theta)=D_{0}(x, \theta)+\left(\Omega^{p} \otimes \mathscr{D}\right)^{+}$.

Proposition 2.3. Let $\omega$ be a global section of $\left(\Omega^{p} \otimes \mathscr{D}\right)^{+}$. Then $\int_{F} \omega$ is exact.

Proof. $\int_{F} \omega=\omega[1]$. Choose a partition of unity $1=\sum \varphi_{\alpha}$, where each $\varphi_{\alpha}$ is a compactly supported section of $\mathscr{A}$ with support in a neighborhood $\mathscr{U}_{\alpha}$ with coordinates $\left(x_{\alpha}, \theta_{\alpha}\right)$. Then $\omega \varphi_{\alpha}=D_{I}\left(x_{\alpha}, \theta_{\alpha}\right) f_{\alpha}^{I}$ where $f_{\alpha}^{0}=0$ and $f_{\alpha}^{I}$ has compact support in $\mathscr{U}_{\alpha}$. It follows that for each $\alpha$ there is a $(p-1)$-form $\eta_{\alpha}$ such that 
$\omega\left[\varphi_{\alpha}\right]=d \eta_{\alpha}$ and $\operatorname{supp} \eta_{\alpha} \subset \operatorname{supp} \varphi_{\alpha}$. Then $\int_{F} \omega=\sum \omega\left[\varphi_{\alpha}\right]=\sum d \eta_{\alpha}$. This last sum is locally finite, so that $\int_{F} \omega=d\left(\sum \eta_{\alpha}\right)$, as desired.

CoRollary 2.4. $\Omega^{p} \otimes \mathscr{D} /\left(\Omega^{p} \otimes \mathscr{D}\right)^{+}=\mathscr{B} e r$, the sheaf of Berezin forms.

Proof. By Proposition 2.3, the Berezin integral as defined in this paper gives a well-defined linear functional on compactly supported sections of $\Omega^{p} \otimes$ $\mathscr{D} /\left(\Omega^{p} \otimes \mathscr{D}\right)^{+}$. It is known that compactly supported sections of $\mathscr{B} e r$ also admit a functional with the same local coordinate expression (2.2) [3]. The integral is nondegenerate in the sense that $\int_{\mathrm{Ber}} \omega \cdot h=0$ for all compactly supported $h$ if and only if $\omega=0$. Thus $\left(\Omega^{p} \otimes \mathscr{D}\right) /\left(\Omega^{p} \otimes \mathscr{D}\right)^{+}$must transform as a Berezin form under change of coordinates.

Since $\left(\Omega^{p} \otimes \mathscr{D}\right)^{+}$is a $\mathscr{D}$-submodule of $\left(\Omega^{p} \otimes \mathscr{D}\right)$, one has

COROLlARY 2.5. $\mathscr{B}$ er has a canonical right $\mathscr{D}$-module structure.

REMARK. This $\mathscr{D}$-module structure is the Lie derivative defined in [3] and discussed further in [4].

3. The transformation properties of $\Omega^{p} \otimes \mathscr{D}$. It is clear from $\S 2$ that one cannot expect to integrate noncompactly supported Berezin forms. The classic example is the supermanifold $C^{\infty}(0,1) \otimes \Lambda\left(\mathbf{R}^{2}\right)$, with even coordinate $x$ and odd coordinates $\theta^{1}$ and $\theta^{2}$. Denote the coordinate Berezin form by $D(x, \theta)$. If $y=x+\theta^{1} \theta^{2}$ and $\eta^{a}=\theta^{a}$, then the superdeterminant, or Berezinian, of $\partial(x, \theta) / \partial(y, \eta)$ is $1 .{ }^{1}$ However

$$
\int D(y, \eta) y=\int_{0}^{1} d y \frac{\partial}{\partial \eta^{2} \partial \eta^{1}}[y]=0,
$$

whereas

$$
\int D(x, \theta)\left(x+\theta^{1} \theta^{2}\right)=\int_{0}^{1} d x \frac{\partial^{2}\left[x+\theta^{1} \theta^{2}\right]}{\partial \theta^{2} \partial \theta^{1}}=1 .
$$

To correct the ambiguity in the Berezin integral, one must pass from $\mathscr{B} e r$ to $\Omega^{p} \otimes \mathscr{D}$. That is,

$$
D_{0}(y, \eta)=\operatorname{Ber}\left(\frac{\partial(y, \eta)}{\partial(x, \theta)}\right) D_{0}(x, \theta)+\sum_{I>0} D_{I}(x, \theta) \Phi^{I}
$$

for some superfunctions $\Phi^{I} .^{2}$ The problem now is to describe this law.

\footnotetext{
${ }^{1}$ Let $B$ be a supercommutative algebra. $\mathrm{Gl}_{p, q}(B)$ is defined to be the group of invertible matrices with coefficients in $B$ having the form $\left(\begin{array}{cc}\alpha & \beta \\ \gamma & \delta\end{array}\right)$, where $\alpha$ is $p \times p$ and has even entries, $\delta$ is $q \times q$ and has even entries, and $\beta$ and $\gamma$ have odd entries. The Berezinian is the one-dimensional representation

$$
\operatorname{Ber}\left(\begin{array}{ll}
\alpha & \beta \\
\gamma & \delta
\end{array}\right)=\operatorname{Det}\left(\alpha-\beta \delta^{-1} \gamma\right) / \operatorname{Det} \delta .
$$

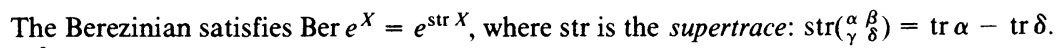

${ }^{2}$ The Jacobian $\partial(y, \eta) / \partial(x, \theta)$ satisfies

$$
\left(\frac{\partial(y, \eta)}{\partial(x, \theta)}\right)_{j}^{i} d x^{j}+\left(\frac{\partial(y, \eta)}{\partial(x, \theta)}\right)_{a}^{i} d \theta^{a}=d y^{i} \quad \text { and }\left(\frac{\partial(y, \eta)}{\partial(x, \theta)}\right)_{j}^{b} d x^{j}+\left(\frac{\partial(y, \eta)}{\partial(x, \theta)}\right)_{a}^{b} d \theta^{a}=d \eta^{b} .
$$
}

Schematically,

$$
\frac{\partial(y, \eta)}{\partial(x, \theta)}=\left(\begin{array}{cc}
\partial y / \partial x & \partial y / \partial \theta \\
\partial \eta / \partial x & -\partial \eta / \partial \theta
\end{array}\right)
$$


Any coordinate system $(x, \theta)$ endows $\mathscr{A}$ with a Z-grading: An element $f \in \mathscr{A}$ has degree 0 if $\partial f / \partial \theta^{a}=0$ for all $a$, and has degree $n+1$ if $\partial f / \partial \theta^{a}$ has degree $n$ for all $a$.

Let $Y$ be a derivation of $\mathscr{A}$. $Y$ will be called degree increasing if for all $j$, $Y\left(\mathscr{N}^{j}\right) \subset \mathscr{N}^{j+1}$, where $\mathscr{N}$ is the nilpotent ideal. (It is sufficient that this hold for $j=0$ and 1.) If $Y$ is even and degree increasing, then $e^{Y}$ defines an automorphism of $\mathscr{A}$.

Proposition 3.1. Let $(x, \theta)$ and $(y, \eta)$ be two coordinate systems for $\mathscr{A}$. Then there exists a unique coordinate system $\left(y_{0}, \eta_{1}\right)$ and a unique even degree-increasing derivation $Y$ such that

(3.1) $\left(y_{0}, \eta_{1}\right)$ and $(x, \theta)$ endow identical Z-gradings to $\mathscr{A}$, and

(3.2) $(y, \eta)=e^{Y}\left(y_{0}, \eta_{1}\right)$.

Proof. Define $y_{0}$ to be the 0 th degree part of $y$ with respect to the $(x, \theta)$ grading and define $\eta_{1}$ to be the first degree part of $\eta$. Then $\left(y_{0}, \eta_{1}\right)$ is a coordinate system. The assignment $y_{0} \rightarrow y, \eta_{1} \rightarrow \eta$ generates an automorphism of $\mathscr{A}$ by Taylor expansion, since $y_{0}-y$ and $\eta_{1}-\eta$ are nilpotent. Call this automorphism $\tau$. Then $1-\tau$ is degree increasing and therefore nilpotent. Define $Y=\log \tau$. The uniqueness follows easily (cf. [7]).

Now write

$$
D_{I}(y, \eta)=D_{J}(x, \theta) \Phi_{I}^{J}
$$

Then $\left(\Phi_{I}^{J}\right)$ is defined by the condition that for all $f$,

$$
(-1)^{|I|} d y \frac{\partial}{\partial y^{I}} \partial_{\eta}[f]=(-1)^{|J|} d x \frac{\partial}{\partial x^{J}} \partial_{\theta}\left[\Phi_{I}^{J} f\right] .
$$

In case $(y, \eta)$ and $(x, \theta)$ endow identical $\mathbf{Z}$-gradings to $\mathscr{A}$, this law reduces essentially to the usual transformation law for differential operators on $M$. We may assume $\mathscr{A}=C^{\infty} \otimes \Lambda\left(\mathbf{R}^{q}\right)$ and that $x^{1}, \ldots, x^{p}$ and $y^{1}, \ldots, y^{q}$ are all sections of $C^{\infty}$. Then $\partial \eta^{a} / \partial \theta^{b} \in C^{\infty}$ for all $a$ and $b$ as well, so that

$$
\partial_{\theta}=\partial_{\eta} \operatorname{Det}\left(d \eta^{b}\left(\frac{\partial}{\partial \theta^{a}}\right)\right)
$$

Therefore $\Phi_{I}^{J}$ is also 0th degree and defined by

$$
\frac{\partial}{\partial y^{I}}=(-1)^{|I|+|J|}\left|\frac{\partial x}{\partial y}\right| \frac{\partial}{\partial x^{J}} \operatorname{Det}\left(d \eta^{b}\left(\frac{\partial}{\partial \theta^{a}}\right)\right) \Phi_{I}^{J} .
$$

In particular

$$
\Phi_{0}^{0}=\operatorname{Det}\left(\frac{\partial y^{j}}{\partial x^{i}}\right) \operatorname{Det}\left(d \theta^{a}\left(\frac{\partial}{\partial \eta^{b}}\right)\right),
$$

which is a special case of the Berezinian transformation law.

Much more interesting is the case in which $(y, \eta)=e^{Y}(x, \theta)$, where $Y$ is a degree-increasing even derivation. Then

$$
\frac{\partial}{\partial y^{I}} \partial_{\eta}=e^{Y} \frac{\partial}{\partial x^{I}} \partial_{\theta} e^{-Y}
$$


Since $e^{Y}=1+$ (degree increasing), one has

$$
D_{I}(y, \eta)=D_{I}(x, \theta) e^{-Y} .
$$

The main result of this section is

THEOREM 3.2. Write

$$
e^{Y}=q A^{J} \frac{\partial}{\partial x^{J}}+\left(\frac{\partial}{\partial \theta^{a}}\right)
$$

where $\left(\partial / \partial \theta^{a}\right)$ is in the left ideal generated by $\partial / \partial \theta^{1}, \ldots, \partial / \partial \theta^{q}$. Then

$$
D_{I}(y, \eta)=D_{I+J}(x, \theta) \operatorname{Ber}\left(\frac{\partial(y, \eta)}{\partial(x, \theta)}\right) g^{J} \text {. }
$$

Proof. For $t \in \mathbf{R}$, let $\left(y_{t}, \eta_{t}\right)=e^{t Y}(x, \theta)$. Let $B_{t}=\operatorname{Ber}\left(\partial(x, \theta) / \partial\left(y_{t}, \eta_{t}\right)\right)$. Let $e^{t Y}=g_{t}^{J}\left(\partial / \partial x^{J}\right)+\left(\partial / \partial \theta^{a}\right)$. The claim is that for all $f$ and $t$,

$$
\left.(-1)^{|I|} \frac{\partial}{\partial y_{t}^{I}} \partial_{\eta_{t}}[f]\right|_{M}=\left.(-1)^{|I|+|J|} \frac{\partial}{\partial x^{I+J}} \partial_{\theta}\left[\frac{1}{B_{t}} g_{t}^{J} f\right]\right|_{M} .
$$

$\partial / \partial y_{t}^{I}$ and $\partial / \partial x^{I}$ agree on $M$ and may be discarded. Also $f$ may be replaced by $B_{t} f$. So the claim is that for all $f$ and $t$

$$
\left.\partial_{\eta_{t}}\left[B_{t} f\right]\right|_{M}=\left.(-1)^{|J|} \frac{\partial}{\partial x^{J}} \partial_{\theta}\left[g_{t}^{J} f\right]\right|_{M}
$$

Let $\sigma_{t}=\partial_{\theta} e^{-t Y} B_{t}$. By (3.4), the left-hand side of (3.5) is $\left.\sigma_{t}[f]\right|_{M}$.

LEMMA 3.3. Let

$$
Y=\lambda^{i} \frac{\partial}{\partial x^{i}}+\gamma^{a} \frac{\partial}{\partial \theta^{a}} \quad \text { and } \quad L=Y+\frac{\partial \lambda^{i}}{\partial x^{i}}-\frac{\partial \gamma^{a}}{\partial \theta^{a}} .
$$

Then $d \sigma_{t} / d t=-\sigma_{t} L$.

Proof.

$$
\begin{aligned}
\frac{d \sigma_{t}}{d t} & =\partial_{\theta}\left(-e^{-t Y} Y B_{t}+e^{-t Y} \frac{d B_{t}}{d t}\right) \\
& =\partial_{\theta} e^{-t Y}\left(\left(\frac{d}{d t}-Y\right)\left[B_{t}\right]-B_{t} Y\right)
\end{aligned}
$$

It must be shown that

$$
\left(\frac{d}{d t}-Y\right)\left[B_{t}\right]=-B_{t}\left(\frac{\partial \lambda^{i}}{\partial x^{i}}-\frac{\partial \gamma^{a}}{\partial \theta^{a}}\right) .
$$

It is convenient to change notation and write

$$
\left(x^{1}, \ldots, x^{p}, \theta^{1}, \ldots, \theta^{q}\right)=\left(x^{1}, \ldots, x^{p+q}\right)
$$

(resp. $y$ ) and $\gamma^{a}=\lambda^{p+a}$. So now $Y=\lambda^{i}\left(\partial / \partial x^{i}\right)$, summing from 1 to $p+q$. The Berezinian is multiplicative, and its derivative at the identity is the supertrace (see 
footnote 1). Therefore

Furthermore

$$
\begin{aligned}
\left(\frac{d}{d t}-Y\right)\left[B_{t}\right] & =\left(\frac{d}{d t}-Y\right)\left[\operatorname{Ber}\left(\frac{\partial x}{\partial y_{t}}\right)\right] \\
& =B_{t} \operatorname{str}\left(\frac{\partial y_{t}}{\partial x} \cdot\left(\frac{d}{d t}-Y\right)\left[\frac{\partial x}{\partial y_{t}}\right]\right) .
\end{aligned}
$$

$$
\begin{aligned}
\frac{d}{d t}\left[\frac{\partial x^{i}}{\partial y_{t}^{j}}\right] & =\frac{d}{d t}\left[e^{t Y} \frac{\partial}{\partial x^{j}} e^{-t Y}\left[x^{i}\right]\right]=\left[Y, \frac{\partial}{\partial y_{t}^{j}}\right]\left[x^{i}\right] \\
& =Y\left[\frac{\partial x^{i}}{\partial y_{t}^{j}}\right]-\frac{\partial}{\partial y_{t}^{j}} Y\left[x^{i}\right] .
\end{aligned}
$$

So

$$
\left(\frac{d}{d t}-Y\right)\left[\frac{\partial x}{\partial y_{t}}\right]_{j}^{i}=-d Y\left[x^{i}\right]\left(\frac{\partial}{\partial y_{t}^{j}}\right)
$$

This gives

$$
\begin{aligned}
\left(\frac{d}{d t}-Y\right)\left[B_{t}\right] & =-B_{t} \operatorname{str}\left(\left(\frac{\partial y_{t}}{\partial x}\right)_{i}^{k} d Y\left[x^{i}\right]\left(\frac{\partial}{\partial y_{t}^{j}}\right)\right) \\
& =-B_{t}\left((-1)^{\left|x^{k}\right|} d y_{t}^{k}\left(\frac{\partial}{\partial x^{i}}\right) d Y\left[x^{i}\right]\left(\frac{\partial}{\partial y_{t}^{k}}\right)\right) .
\end{aligned}
$$

Switch the two factors and remember the sign changes:

$$
\begin{aligned}
& =-B_{t}\left((-1)^{\left|x^{i}\right|} d Y\left[x^{i}\right]\left(\frac{\partial}{\partial y_{t}^{k}}\right) d y_{t}^{k}\left(\frac{\partial}{\partial x^{i}}\right)\right) \\
& =-B_{t}\left((-1)^{\left|x^{i}\right|} d Y\left[x^{i}\right]\left(\frac{\partial}{\partial x^{i}}\right)\right) .
\end{aligned}
$$

Since

$$
\begin{gathered}
\frac{\partial x^{i}}{\partial x^{j}}=(-1)^{\left|x^{i}\right|\left|x^{j}\right|} d x^{i}\left(\frac{\partial}{\partial x^{j}}\right)=(-1)^{\left|x^{i}\right|\left|x^{j}\right|} \delta_{j}^{i}, \\
Y\left[x^{i}\right]=(-1)^{\left|x^{i}\right|} \lambda^{i}, \text { so } \\
\left(\frac{d}{d t}-Y\right)\left[B_{t}\right]=-B_{t} \cdot d \lambda^{i}\left(\frac{\partial}{\partial x^{i}}\right)=(-1)^{\left|x^{i}\right|} B_{t} \frac{\partial \lambda^{i}}{\partial x^{i}},
\end{gathered}
$$

which is what was claimed.

Now let

$$
\rho_{t}=(-1)^{|J|} \partial_{\theta} \frac{\partial}{\partial x^{J}} g_{t}^{J}
$$

The proof of Theorem 3.2 will be complete once it is shown that $d \rho_{t} / d t=-\rho_{t} L$. Observe that if $f$ is compactly supported, then

$$
\int D_{0}(x, \theta) \frac{\partial f}{\partial x^{i}}=\int D_{0}(x, \theta) \frac{\partial f}{\partial \theta^{a}}=0 .
$$


Thus

$$
\int D_{0}(x, \theta) \partial_{\theta}[h] f=(-1)^{(|h|+1) q} \int D_{0}(x, \theta) h \partial_{\theta} f
$$

if $h f$ is compactly supported. Then

$$
\begin{aligned}
\int D_{0}(x, \theta) \rho_{t}[h] f & =\int D_{0}(x, \theta)(-1)^{|J|} \frac{\partial}{\partial x^{J}} \partial_{\theta}\left[g_{t}^{J} h\right] f \\
& =\int D_{0}(x, \theta) \partial_{\theta}\left[g_{t}^{J} h\right] \frac{\partial}{\partial x^{J}} f \\
& =(-1)^{(|h|+1) q} \int D_{0}(x, \theta) h g_{t}^{J} \frac{\partial}{\partial x^{J}} \partial_{\theta} f \\
& =(-1)^{(|h|+1) q} \int D_{0}(x, \theta) h e^{t Y} \partial_{\theta} f .
\end{aligned}
$$

Therefore

$$
\begin{aligned}
\frac{d}{d t} \int D_{0}(x, \theta) \rho_{t} & {[h] f=(-1)^{(|h|+1) q} \int D_{0}(x, \theta) h Y e^{t Y} \partial_{\theta} f } \\
& =(-1)^{(|h|+1) q} \int D_{0}(x, \theta) h\left(\lambda^{i} \frac{\partial}{\partial x^{i}}+\gamma^{a} \frac{\partial}{\partial \theta^{a}}\right) e^{t Y} \partial_{\theta} f \\
& =(-1)^{(|h|+1) q} \int D_{0}(x, \theta)\left(-\frac{\partial\left(h \lambda^{i}\right)}{\partial x^{i}}-(-1)^{(1+|h|)} \frac{\partial\left(h \gamma^{a}\right)}{\partial \theta^{a}}\right) e^{t Y} \partial_{\theta} f \\
& =(-1)^{(|h|+1) q} \int D_{0}(x, \theta)\left(-\frac{\partial\left(\lambda^{i} h\right)}{\partial x^{i}}+\frac{\partial\left(\gamma^{a} h\right)}{\partial \theta^{a}}\right) e^{t Y} \partial_{\theta} f \\
& =-\int D_{0}(x, \theta) \rho_{t} L[h] f .
\end{aligned}
$$

That is,

$$
\int D_{0}(x, \theta) \frac{d \rho_{t}}{d t}[h] f=-\int D_{0}(x, \theta) \rho_{t} L[h] f
$$

for all compactly supported $f$. So $d \rho_{t} / d t=-\rho_{t} L$, which completes the proof of Theorem 3.2.

To illustrate, return to the example

$$
\int D_{0}(y, \eta)\left(f_{0}(y)+\eta^{1} \eta^{2} f_{1}(y)\right)
$$

with $y$ ranging over $(0,1)$. Set $y=x+\theta^{1} \theta^{2} g(x)$ and $\eta^{a}=\theta^{a}$. Then

$$
\frac{\partial(y, \eta)}{\partial(x, \theta)}=\left(\begin{array}{ccc}
1+\theta^{1} \theta^{2} g^{\prime}(x) & \theta^{2} g & -\theta^{1} g \\
0 & 1 & 0 \\
0 & 0 & 1
\end{array}\right) .
$$

So

$$
\operatorname{Ber}\left(\frac{\partial(y, \eta)}{\partial(x, \theta)}\right)=1+\theta^{1} \theta^{2} g^{\prime}(x)
$$


Also $(y, \eta)=e^{Y}(x, \theta)$, where $Y=\theta^{1} \theta^{2} g(x) \partial / \partial x$. Then

$$
\begin{aligned}
D_{0}(y, \eta)= & D_{0}(x, \theta)\left(1+\theta^{1} \theta^{2} g^{\prime}(x)\right) \\
& +D_{1}(x, \theta)\left(1+\theta^{1} \theta^{2} g^{\prime}(x)\right)\left(\theta^{1} \theta^{2} g(x)\right) \\
= & D_{0}(x, \theta)\left(1+\theta^{1} \theta^{2} g^{\prime}(x)\right)+D_{1}(x, \theta) \theta^{1} \theta^{2} g(x) .
\end{aligned}
$$

Then

$$
\int D_{0}(y, \eta)\left(f_{0}(y)+\eta^{1} \eta^{2} f_{1}(y)\right)=\int d y f_{1}(y)=\int d x f_{1}(x)
$$

while

$$
\begin{aligned}
\int\left[D_{0}(x, \theta)\left(1+\theta^{1} \theta^{2} g^{\prime}\right)+D_{1}(x, \theta) \theta^{1} \theta^{2} g\right]\left[f_{0}\left(x+\theta^{1} \theta^{2} g\right)+\theta^{1} \theta^{2} f_{1}\left(x+\theta^{1} \theta^{2} g\right)\right] \\
=\int\left[D_{0}(x, \theta)\left(1+\theta^{1} \theta^{2} g^{\prime}\right)+D_{1}(x, \theta) \theta^{1} \theta^{2} g\right]\left[f_{0}+f_{0}^{\prime} g \theta^{1} \theta^{2}+f_{1} \theta^{1} \theta^{2}\right] \\
=\int D_{0}(x, \theta) \theta^{1} \theta^{2}\left(f_{0} g^{\prime}+f_{0}^{\prime} g+f_{1}\right)+D_{1}(x, \theta) \theta^{1} \theta^{2} g f_{0} \\
=\int D_{0}(x, \theta) \theta^{1} \theta^{2}\left(g^{\prime}(x) f_{0}(x)+f_{0}^{\prime}(x) g(x)+f_{1}(x)-\frac{d}{d x}\left(g(x) f_{0}(x)\right)\right) \\
=\int d x f_{1}(x),
\end{aligned}
$$

as desired.

4. Stokes' theorem. $\Omega^{p} \otimes \mathscr{D}$ belongs to the complex $\left(\Omega_{M}^{*} \otimes_{\mathscr{A}} \mathscr{D}, d^{\prime}\right)$, where $d^{\prime}$ is defined in such a way that it is right $\mathscr{A}$ linear and $\int_{F}$ is a chain map: $d^{\prime} \omega[f]=$ $d(\omega[f])$.

PROPOSITION 4.1. The cohomology sheaf of $\left(\Omega^{*} \otimes \mathscr{D}, d^{\prime}\right)$ is

$$
\mathscr{H}^{k}\left(\Omega^{\cdot} \otimes \mathscr{D}, d^{\prime}\right)= \begin{cases}0, & k \leqslant p, \\ \mathscr{B}_{\text {er }}, & k=p .\end{cases}
$$

Proof. This is essentially given in [4] at the end of $\$ 1.4$. There $\Omega$ is replaced by the sheaf of differential forms on the supermanifold rather than on the manifold. In the present context, $\mathscr{H}^{p}\left(\Omega^{\circ} \otimes \mathscr{D}, d^{\prime}\right)=\mathscr{B}$ er is almost immediate, since $\mathscr{B}$ er is explicitly $\Omega^{*} \otimes \mathscr{D} /\left(\Omega^{*} \otimes \mathscr{D}\right)^{+}$. One then proves directly that $\mathscr{H}^{p-1}\left(\Omega^{*} \otimes \mathscr{D}, d^{\prime}\right)=0$, and the rest follows by induction on $p$.

Let $(M, \mathscr{A}) \stackrel{\Phi}{\rightarrow}(N, \mathscr{B})$ be a morphism of supermanifolds. $\Phi$ consists of an underlying map $M \stackrel{\tilde{\varphi}}{\rightarrow} N$ and a sheaf morphism $\tilde{\varphi}^{-1} \mathscr{B} \stackrel{\varphi}{\rightarrow} \mathscr{A}$. Given $m \in M$ and $\left.\omega \in \Omega_{N}^{k} \otimes \mathscr{D}_{\mathscr{B}}\right|_{\tilde{\varphi}(m)}$ and $\left.f \in \mathscr{A}\right|_{m}$, the pullback $\left.\Phi^{*} \omega\right|_{m}$ is defined by $\Phi^{*} \omega[f]=$ $\tilde{\varphi}^{*}(\omega[g])$, where $\varphi(g)=f$. Thus $\Phi^{*} \omega$ is defined only if $\operatorname{ker} \varphi \subseteq \operatorname{ker}\left(\tilde{\varphi}^{*} \circ \omega\right)$, and even then it is defined only as an operator on the range of $\varphi$.

To define supermanifold with boundary, two modifications of the definition of supermanifold are needed. First, $M$ now has a boundary $\partial M \stackrel{\iota}{\hookrightarrow} M$. Second, there is a supermanifold $\partial \mathscr{A}$ over $\partial M$ and a morphism $\left.\mathscr{A}\right|_{\partial M} \stackrel{\iota}{\rightarrow} \partial \mathscr{A}$. 
Stokes' theorem follows immediately from the usual Stokes' theorem. Indeed

$$
\begin{aligned}
\int_{(M, \mathscr{A})} d^{\prime} \omega & =\int_{M} d(\omega[1])=\int_{\partial M} \omega[1] \\
& =\int_{\partial M} \iota^{*} \omega[1]=\int_{(\partial M, \partial \mathscr{A})} \iota^{*} \omega .
\end{aligned}
$$

Note that no reference is made to the odd dimension of $\partial \mathscr{A}$. The choice $\operatorname{dim} \partial \mathscr{A}=(p-1, q)$ is not forced, but Stokes' theorem offers two arguments in favor of such a choice. First, if the map $\left.\mathscr{A}\right|_{\partial M} \stackrel{\iota}{\rightarrow} \partial \mathscr{A}$ is surjective, then the pullback, $\iota^{*} \omega$, when it is defined, will be defined on all of $\partial \mathscr{A}$. Without this, the results of the previous sections are unavailable. This forces the odd dimension of $\partial \mathscr{A}$ to be at most $q$. Then $q$ itself is preferred so that ker $\iota$ will be as small as possible.

\section{REFERENCES}

1. F. A. Berezin, The method of second quantization, Pure and Appl. Physics, vol. 24, Academic Press, New York, 1966.

2. B. Kostant, Graded manifolds, graded Lie theory and prequantization, Differential Geometric Methods in Mathematical Physics (Proc. Sympos. Univ. Bonn, Bonn, 1975), Lecture Notes in Math., vol. 570, Springer, Berlin, 1977, pp. 177-306.

3. D. A. Leites, Introduction to the theory of supermanifolds, Russian Math. Surveys 35 (1980), no. 1, $1-64$.

4. I. B. Penkov, D-modules on supermanifolds, Invent. Math. 71 (1983), 501-512.

5. J. M. Rabin, The Berezin integral as contour integral, Univ. of Chicago preprint, EF1 84/4.

6. A. Rogers, Consistent superspace integration, J. Math. Phys. 26 (1985), 385-392.

7. M. Rothstein, Deformations of complex supermanifolds, Proc. Amer. Math. Soc. 95 (1985), 255-260.

DePartment of Mathematics, University of Washington, Seattle, Washington 98195

Current address: School of Mathematics, Institute for Advanced Study, Princeton, New Jersey 08540 